\title{
Optogenetic manipulation of cGMP highlights PDE5 as the predominant cGMP-hydrolyzing PDE in megakaryocytes
}

Yujing Zhang ${ }^{1, *}$, Pascal Benz ${ }^{1, *}$, Daniel Stehle ${ }^{2}$, Shang Yang ${ }^{3}$, Hendrikje Kurz ${ }^{1}$, Susanne Feil ${ }^{2}$, Georg Nagel ${ }^{3}$, Robert Feil ${ }^{2}$, Shiqiang Gao ${ }^{3, \star *}$, Markus Bender ${ }^{1, * *}$

${ }^{1}$ Institute of Experimental Biomedicine - Chair I, University Hospital and Rudolf Virchow Center, Würzburg, Germany

${ }^{2}$ Interfakultäres Institut für Biochemie, University of Tübingen, Tübingen, Germany

${ }^{3}$ Department of Neurophysiology, Institute of Physiology, Biocenter, University of Würzburg, Germany.

${ }^{*}$ contributed equally

${ }^{* *}$ Correspondence to:

Markus Bender: Bender_M1@ukw.de

Shiqiang Gao: $\quad$ gao.shiqiang@uni-wuerzburg.de 


\section{Abstract}

Cyclic guanosine monophosphate (cGMP) signalling plays a fundamental role in many cell types including platelets. cGMP has been implicated in platelet formation, but mechanistic detail about its spatiotemporal regulation in megakaryocytes (MKs) is lacking. We expressed a photo-activated guanylyl cyclase, Blastocladiella emersonii Cyclase opsin (BeCyclop), after viral-mediated gene transfer in bone marrow (BM)-derived MKs to precisely light-modulate cGMP levels. BeCyclop-MKs showed a significantly increased cGMP concentration after illumination, which was strongly dependent on phosphodiesterase (PDE) 5 activity. This finding was corroborated by real-time imaging of cGMP signals which revealed that pharmacological PDE5 inhibition also potentiated nitric oxide (NO) triggered cGMP generation in BM MKs. In summary, we established for the first time optogenetics in primary MKs and identified PDE5 as the predominant PDE regulating cGMP levels in MKs. These findings also demonstrate that optogenetics allows for the precise manipulation of MK biology.

\section{Introduction}

Binding of nitric oxide (NO) to guanylyl cyclases increases the cGMP formation. cGMP is a key intracellular signalling molecule in many cell types and tissues and exerts multiple cellular effects via its downstream effectors, such as protein kinase $G(P K G)$ or cyclic nucleotide-gated (CNG) channels (1). The cGMP signalling pathway is long known for its critical role in the maintenance of cardiovascular homeostasis. Fluorescent cGMP biosensors have emerged as powerful tools for the sensitive analysis of cGMP pathways at the single-cell level (2), and methods that allow for precise manipulation of cGMP levels by light are being developed (3). The NO/cGMP/PKG pathway is highly expressed in platelets and its activation has been linked to platelet inhibition (4). Analysis of spatiotemporal dynamics of platelet cGMP using cGMP sensor mice revealed that CGMP generation is increased in shear-exposed platelets at the periphery of a thrombus thereby limiting thrombus growth (5). Cellular cGMP levels are regulated by phosphodiesterases (PDEs), which catalyse the hydrolysis of the $3^{\prime}$ phosphate bond of cGMP to generate 5' GMP. Platelets express PDE2, 3 and 5, of which each is able to degrade cGMP (4-7). Bone marrow (BM) megakaryocytes (MKs) are the immediate progenitor cells of blood platelets (8). Studies on MKs derived from mouse fetal liver cells showed that PDE3A, PDE4A1 and PDE5 are detectable in maturing MKs, while PDE2A is found in the nonMK fraction, and that in vitro platelet release is enhanced by cGMP (9). However, the spatiotemporal regulation of cGMP signals by PDEs in MKs has not been addressed due to the lack of appropriate tools. Therefore, we expressed a light-sensitive guanylyl cyclase in primary BM-derived MKs to tightly manipulate cGMP levels (3). We demonstrate that the cGMP concentration in these MKs can be increased upon illumination, which rapidly declines in the dark caused by PDE5 activity. 


\section{Results and Discussion}

Concentration-dependent increase of cGMP in BM-derived MKs was measured when cells had been incubated with riociguat, a pharmacological stimulator of the NO-sensitive guanylyl cyclase (Fig. 1A). To tightly manipulate cGMP levels, the photoactivated guanylyl cyclase opsin from Blastocladiella emersonii, BeCyclop (Fig. 1B), was expressed in BM-derived MKs after viral transduction, which allows light-triggered cGMP increase as shown in heterologous cells and Caenorhabditis elegans (3). Localization of YFP-BeCyclop in the membrane system of BM-derived MKs was confirmed by confocal microscopy (Fig. 1C). Illumination (green light: $520 \mathrm{~nm}, 5 \mathrm{~min})$ of YFP-BeCyclop MKs resulted in a significant increase in cGMP $(3.25 \pm 0.43$ $\mathrm{pmol} / \mathrm{mL})$ as compared to samples in the dark $(0.76 \pm 0.06 \mathrm{pmol} / \mathrm{mL})$ and untransduced cells (no light: $0.95 \pm 0.09 \mathrm{pmol} / \mathrm{mL}$; light: $0.84 \pm 0.02 \mathrm{pmol} / \mathrm{mL}$ ) (Fig. 1D). These data demonstrate that it is possible to modulate intracellular cGMP in primary MKs with optogenetic approaches. Stimulation with $5 \mu \mathrm{M}$ riociguat resulted in a similar cGMP increase as observed after 5 min illumination of YFP-BeCyclop MKs (Fig. 1D). However, it has to be noted that direct comparison cannot be made since the transduction efficiency of YFP-BeCyclop was only about $\sim 30 \%$ in MKs suggesting even higher cGMP values per cell after illumination. Interestingly, a rapid decrease of the intracellular cGMP concentration close to the baseline level was observed within 3 min after illumination, pointing to a high cGMP-hydrolyzing PDE activity in MKs (Fig. 1E). Copy number analysis of the murine platelet proteome revealed a high PDE5 expression in murine platelets (10). Therefore, cells were pretreated with the specific PDE5 inhibitor tadalafil, which resulted in a significant but only subtle cGMP increase (DMSO: $1.02 \pm 0.15 \mathrm{pmol} / \mathrm{mL}$; tadalafil: $1.34 \pm 0.09 \mathrm{pmol} / \mathrm{mL}$; Fig. 1F). However, cGMP levels were 6.9fold increased when pretreated with tadalafil and 5 min illuminated $(20.45 \pm 4.3 \mathrm{pmol} / \mathrm{mL})$, as compared to illumination without tadalafil pretreatment $(2.95 \pm 0.45 \mathrm{pmol} / \mathrm{mL})$. These data strongly suggest that PDE5 limits the cGMP concentration increase in MKs. Furthermore, inhibition of PDE5 resulted in less cGMP decrease to $10 \pm 1.5 \mathrm{pmol} / \mathrm{mL}$ in $3 \mathrm{~min}$ after illumination (Fig. 1F), again comfirming that PDE5 degrades CGMP in MKs. In agreement with our findings with tadalafil, illuminated MKs preincubated with other PDE5 inhibitors (vardenafil: PDE5, 6; sildenafil: PDE5, 6) also significantly increased cGMP concentration compared to illuminated YFP-BeCyclop MKs without inhibitor (Fig. 1G,H), whereas non-PDE5 inhibitors did not elevate intracellular cGMP (vinpocetine: PDE1; EHNA: PDE2; BAY60-7550: PDE2; milrinone: PDE3, 4; TAK-063: PDE10; Fig. 1G). Interestingly, also the broad-spectrum PDE inhibitors zaprinast (PDE5, 6, 9, 10, 11) and IBMX (PDE1, 2, 3, 4, 5, 7, 11) had no or only minor effects, respectively (Fig. 1G).

To demonstrate the high specificity of BeCyclop, MKs expressing YFP-BeCyclop were either stimulated with the adenylyl cyclase activator forskolin or illuminated and subsequently, cyclic adenosine monophosphate (cAMP) was determined. While forskolin significantly increased the 
cAMP concentration in MKs, illumination had no effect on cAMP concentration, demonstrating that BeCyclop cannot produce or influence cAMP (Fig. 1I).

Next, we used MK/platelet-specific cGMP sensor mice to spatiotemporally visualize cGMP dynamics in MKs ex vivo (Fig. 2A) $(5,11)$. Isolated femurs with externalized bone marrow were superfused with $1 \mathrm{~mL} / \mathrm{min}$ imaging buffer. In contrast to static cell culture experiments, this method allows to rapidly add and remove the pharmacological compounds within seconds similar to optogenetic experiments. Application of the NO donor diethylamine NONOate (DEA/NO) increased cGMP in BM MKs (Fig. 2B,C). Similar to the optogenetic results, the PDE5-specific inhibitor tadalafil alone slowly increased cGMP and significantly augmented the NO-induced cGMP signal in MKs (Fig. 2B,C). These data strongly suggest that BM MKs in their native environment express a functional NO/cGMP/PDE5 signalling pathway that can be pharmacologically enhanced with PDE5 inhibitors.

In summary, we established to the best of our knowledge the first time optogenetics in primary MKs. By expressing the photo-activated guanylyl cyclase, BeCyclop, we tightly controlled cGMP levels in MKs by light. We identified PDE5 as the major PDE, counteracting cGMP levels in BM-derived MKs, and could verify our optogenetic results by cGMP imaging in BM MKs ex vivo using cGMP sensor mice. Our findings are corroborated by research on mouse fetal liver cell (FLC)-derived MKs showing PDE5 expression is highest in mature FLC-derived MKs (9). Our study shows that optogenetics in MKs allows to light-modulate the function of key signalling molecules thereby possibly identifying novel regulatory mechanisms of $\mathrm{MK}$ maturation and platelet production. However, our study has also limitations as the transduction efficiency of MKs was only about $30 \%$. We analysed the complete MK fraction since complicated sorting of mature MKs (cell diameter of approx. $35 \mu \mathrm{m}$ ) most likely leads to lightactivation before starting the experiment. Thus, our data underestimate the light-induced increase of cGMP in single MKs expressing YFP-BeCyclop due to the untransduced cell fraction. Further studies are required using MK/platelet-specific optogenetic mouse lines to spatiotemporally light-manipulate molecules or proteins in MKs and platelets in vivo. The finding that BM MK express PDE5 also informs future therapeutic strategies to increase cGMP concentrations and potentially modulate platelet biogenesis with clinically used PDE5 inhibitors. 


\section{Materials and Methods}

Animals. MK/platelet-specific cGMP sensor mice (cGi500-L2 $\left.2^{f / f f l} ; \mathrm{Pf} 4-\mathrm{Cre}^{\mathrm{tg} / \mathrm{+}(12)}\right)$ were used to visualize cGMP signals in MKs in real-time $(5,11)$.

Reagents. Riociguat (Selleck Chemicals), tadalafil (Sigma-Aldrich/Supelco), DMSO, forskolin (Sigma-Aldrich), vinpocetine, EHNA (Enzo Life Sciences), BAY60-7550, TAK-063 (Cayman Chemical), milrinone, vardenafil, zaprinast (Santa Cruz Biotechnology), sildenafil (Merck), IBMX (Thermo Fisher Scientific), DEA/NO (Axxora).

BM-derived MKs. BM cells were obtained from femur and tibia of C57BL/6 mice by flushing, and lineage depletion was performed using an antibody cocktail of anti-mouse CD3, clone 17A2; anti-mouse Ly-6G/Ly-6C, clone RB6-8C5; anti-mouse CD11b, clone M1/70; anti-mouse CD45R/B220, clone RA3-6B2; anti-mouse TER-119/Erythroid cells, clone Ter-119 (1.5 $\mu \mathrm{g}$ of each antibody per mouse, Biolegend) and magnetic beads (Dynabeads $®$ Untouched Mouse CD4 Cells, Invitrogen). Lineage-negative (Lin-) cells were cultured in DMEM medium (supplemented with $4 \mathrm{mM}$ L-glutamine, $100 \mathrm{U} \mathrm{mL}-1$ penicillin, $50 \mathrm{mg} \mathrm{mL}-1$ streptomycin) containing $1 \%$ of recombinant TPO (homemade) and $100 \mathrm{U} \mathrm{mL}-1$ recombinant Hirudin (Hyphen Biomed) at $37^{\circ} \mathrm{C}$ under $5 \% \mathrm{CO}_{2}$.

Expression of YFP-BeCyclop in MKs. YFP-BeCyclop DNA was cloned into the murine stem cell virus (MSCV) vector and transfected into 293T cells. Viral supernatant was collected and BM-derived cells were infected on day 1 (13). A bovine serum albumin density gradient was used at culture day 3 to separate MKs from non-MK cells. Experiments were performed on day 4.

Illumination and determination of cGMP concentration. Cell density was adjusted to $5 \mathrm{x}$ $10^{4}$ cells $/ \mathrm{mL}$. If needed, samples were preincubated with agonist or inhibitor for $10 \mathrm{~min}$ at room temperature. Subsequently, samples were kept in the dark or illuminated with light $(520 \mathrm{~nm}$, $20 \mu \mathrm{W} / \mathrm{mm}^{2}$ ) for $5 \mathrm{~min}$ at room temperature and lysed either immediately or after $3 \mathrm{~min}$ in the dark with $0.1 \mathrm{M}$ hydrogen-chloride $(\mathrm{HCl})$. Concentrations of cGMP or cAMP were measured by DetectX Direct cyclic GMP or AMP Enzyme Immunoassay Kit, respectively, and analysed by the optical density at $450 \mathrm{~nm}$ with a Thermo Scientific ${ }^{\mathrm{TM}}$ Multiskan microplate spectrophotometer.

Real-time cGMP imaging in MKs ex vivo. Femurs and tibias were dissected from MK/platelet-specific cGMP sensor mice (cGi500-L2 f//fl; Pf4-Cre ${ }^{t g /+}(12)$ ). Bone tissue was carefully removed with scissor and forceps on one side to give access to the bone marrow. 
Opened bones were placed in a superfusion chamber (RC 26, Warner Instruments), mounted with a Slice Hold-Down (SHD 26H/10, Warner Instruments) and continuously superfused with imaging buffer (140 mM NaCl, $5 \mathrm{mM} \mathrm{KCl}, 1.2 \mathrm{mM} \mathrm{MgCl} 2,2 \mathrm{mM} \mathrm{CaCl} 2,5 \mathrm{mM} \mathrm{HEPES}$ and 10 $\mathrm{mM}$ D-glucose; $\mathrm{pH}=7.4$ ) with or without added drugs. Real-time FRET/cGMP imaging was performed by recording CFP and YFP fluorescence as described in detail elsewhere (14). The relative CFP/YFP ratio change (black trace in the respective graph; referred to as $R$ (cGMP)) correlates with the cGMP concentration change.

Data analysis. Results are shown as mean \pm SEM. Statistical significance was assessed by an unpaired t-test with GraphPad Prism software. P-values $<0.05$ were considered significant $\left({ }^{* * *}<0.0001 ;{ }^{* * *}<0.001 ;{ }^{* *} 0.001\right.$ to $0.01 ;{ }^{*} 0.01$ to 0.05$)$. ns: non-significant. Statistical analysis of cGMP sensor imaging data was performed with Origin 2019 (OriginLab, Northampton, MA, USA). Since data sets were not normally distributed, statistical differences were analysed non-parametrically by Mann-Whitney $U$ Test.

\section{Data availability}

All study data are in the article.

\section{Acknowledgments}

The authors thank Sarah Seidel for the help with experiments. This work was supported by TR240 grant with project number 374031971 of the Deutsche Forschungsgemeinschaft (DFG; German Research Foundation).

\section{References}

1. A. Friebe, P. Sandner, A. Schmidtko, cGMP: a unique 2nd messenger molecule recent developments in cGMP research and development. Naunyn Schmiedebergs Arch Pharmacol 393, 287-302 (2020).

2. R. Feil, M. Lehners, D. Stehle, S. Feil, Visualising and understanding cGMP signals in the cardiovascular system. Br J Pharmacol 10.1111/bph.15500 (2021).

3. S. Gao et al., Optogenetic manipulation of cGMP in cells and animals by the tightly light-regulated guanylyl-cyclase opsin CyclOp. Nat Commun 6, 8046 (2015).

4. U. Walter, S. Gambaryan, cGMP and cGMP-dependent protein kinase in platelets and blood cells. Handb Exp Pharmacol 10.1007/978-3-540-68964-5_23, 533-548 (2009).

5. L. Wen et al., A shear-dependent NO-cGMP-cGKI cascade in platelets acts as an autoregulatory brake of thrombosis. Nat Commun 9, 4301 (2018).

6. P. Gresele, S. Momi, E. Falcinelli, Anti-platelet therapy: phosphodiesterase inhibitors. Br J Clin Pharmacol 72, 634-646 (2011).

7. M. T. Rondina, A. S. Weyrich, Targeting phosphodiesterases in anti-platelet therapy. Handb Exp Pharmacol 10.1007/978-3-642-29423-5_9, 225-238 (2012).

8. K. R. Machlus, J. E. Italiano, Jr., The incredible journey: From megakaryocyte development to platelet formation. J Cell Biol 201, 785-796 (2013).

9. A. J. Begonja et al., Differential roles of cAMP and cGMP in megakaryocyte maturation and platelet biogenesis. Exp Hematol 41, 91-101 e104 (2013). 
10. M. Zeiler, M. Moser, M. Mann, Copy number analysis of the murine platelet proteome spanning the complete abundance range. Mol Cell Proteomics 13, 3435-3445 (2014).

11. M. Thunemann et al., Transgenic mice for cGMP imaging. Circ Res 113, 365-371 (2013).

12. R. Tiedt, T. Schomber, H. Hao-Shen, R. C. Skoda, Pf4-Cre transgenic mice allow the generation of lineage-restricted gene knockouts for studying megakaryocyte and platelet function in vivo. Blood 109, 1503-1506 (2007).

13. M. Bender et al., Microtubule sliding drives proplatelet elongation and is dependent on cytoplasmic dynein. Blood 125, 860-868 (2015).

14. D. Stehle et al., Novel soluble guanylyl cyclase activators increase glomerular cGMP, induce vasodilation and improve blood flow in the murine kidney. $\mathrm{Br} J$ Pharmacol 10.1111/bph.15586 (2021). 
A

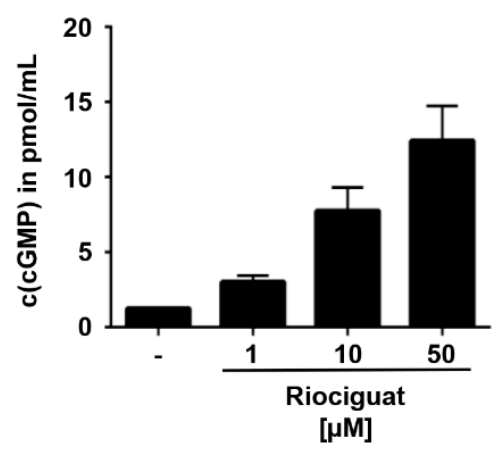

D

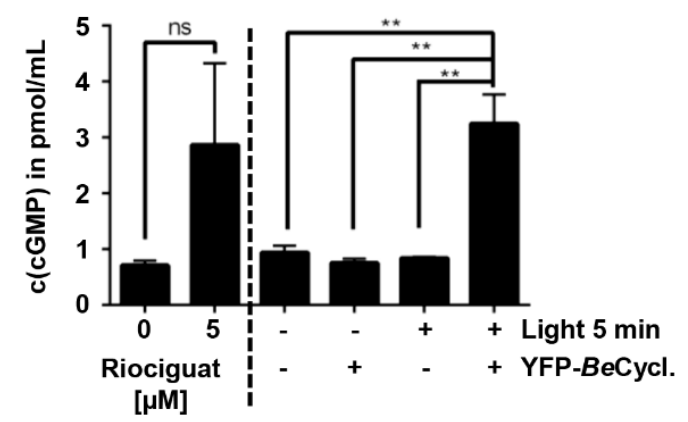

B

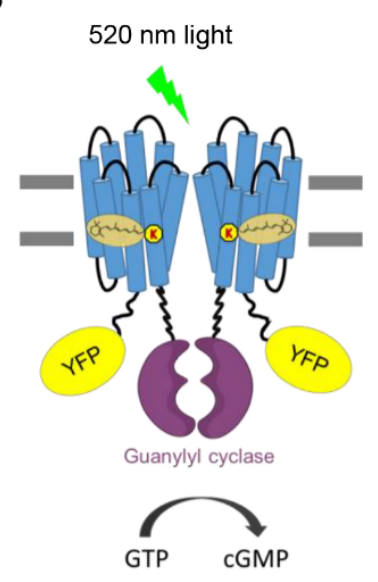

C

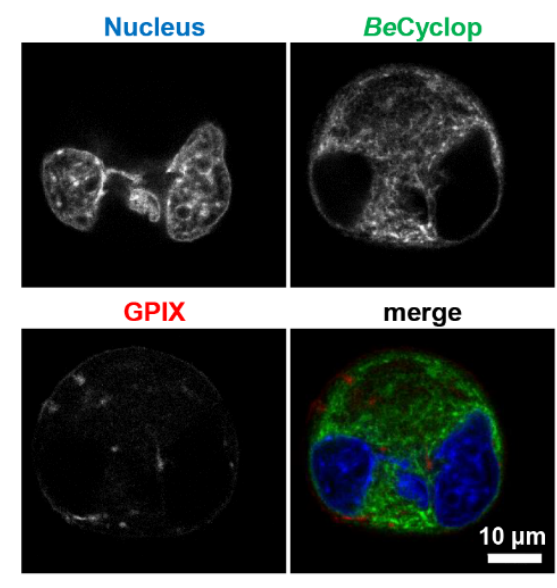

\section{E}

G

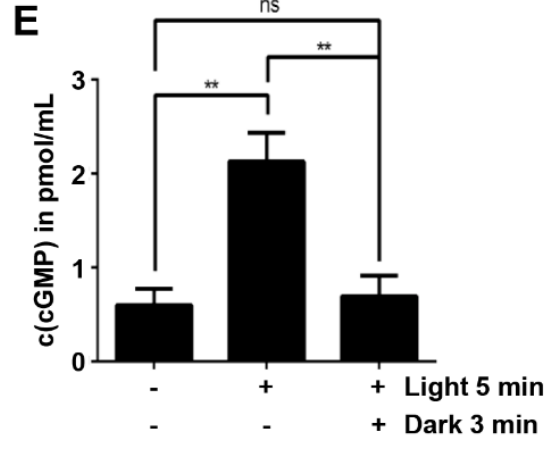

$\mathbf{F}$

$\square 0$ min light $\square 5$ min light $\square 5$ min light/

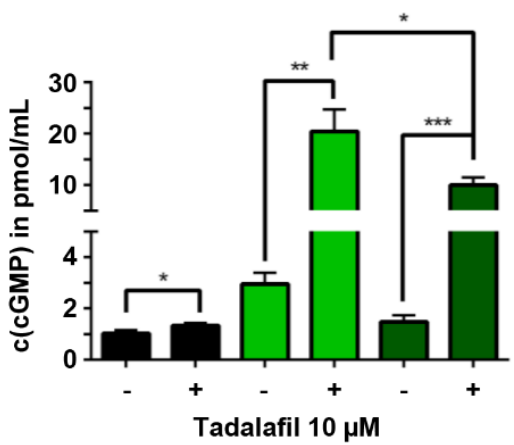

H

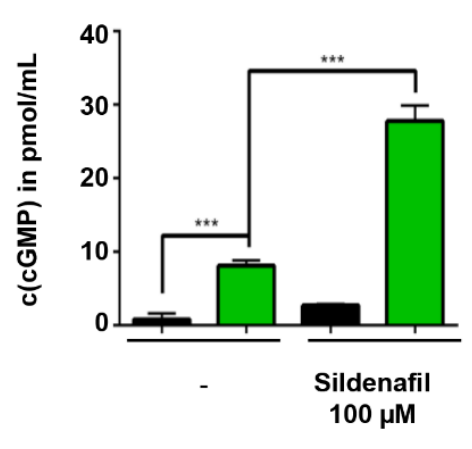

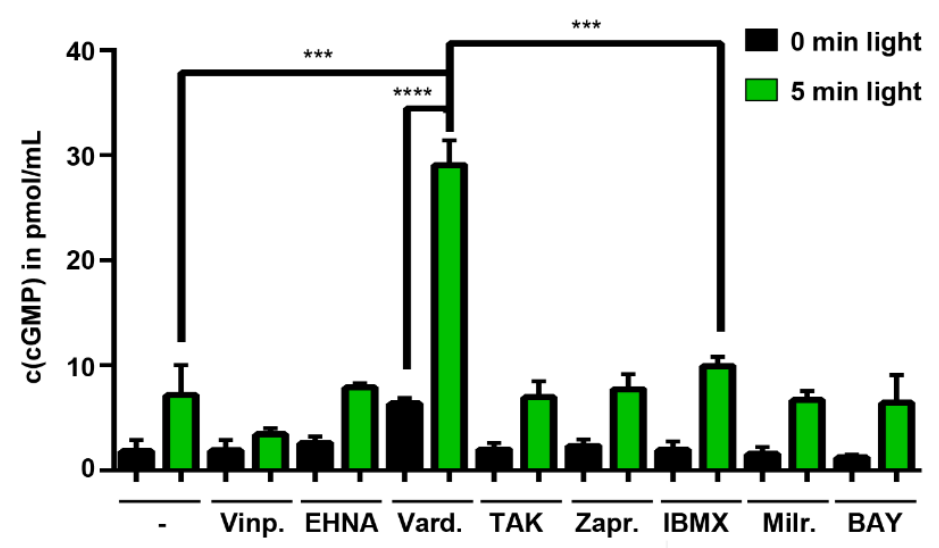

I

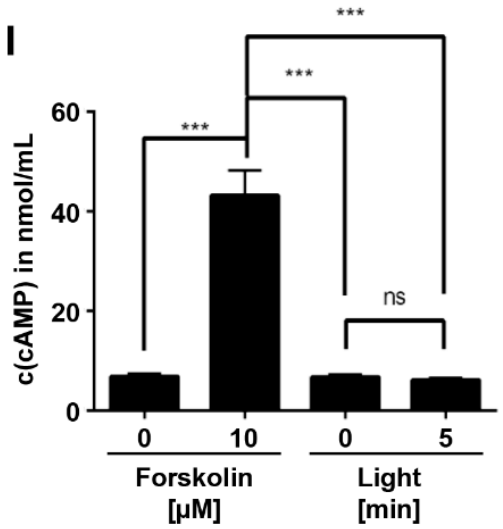


Figure 1. PDE5 regulates optogenetically-increased cGMP levels in MKs. (A) BM-derived MKs were incubated for 10 min with 1,10 , or $50 \mu \mathrm{M}$ riociguat. DMSO was used as vehicle control. ( $n=3$ ). (B) A schematic model of the light-gated guanylyl cyclase, BeCyclop. (C) Representative images (microscope: Leica SP8) of YFP-BeCyclop expression in BM-derived MKs. MK/platelet-specific membrane protein glycoprotein (GP) IX. (D) BM-derived YFPBeCyclop-expressing MKs were illuminated with green light $(520 \mathrm{~nm})$ for $5 \mathrm{~min}$ and immediately lysed. Untransduced cells or no illumination served as controls. $(n=3$, representative for 2 independent experiments). (E) YFP-BeCyclop-expressing MKs were illuminated with green light $(520 \mathrm{~nm})$ for $5 \mathrm{~min}$ and immediately lysed or after $3 \mathrm{~min}$ in the dark. (F) BM-derived MKs were preincubated with tadalafil for $10 \mathrm{~min}$ prior to $5 \mathrm{~min}$ illumination. Cells were either lysed immediately or after 3 min before measuring cGMP concentration. $(n=3$, representative for 2 independent experiments). (G, H) BM-derived MKs were preincubated with different PDE inhibitors for 10 min prior to illumination. Cells were either lysed immediately or after $3 \mathrm{~min}$ in the dark before measuring cGMP concentration. DMSO control in (G), $\mathrm{H}_{2} \mathrm{O}$ control in $(\mathrm{H})$. ( $\mathrm{n}=3$ ). Vinpocetine (Vinp.): $20 \mu \mathrm{M}$, EHNA: $10 \mu \mathrm{M}$, vardenafil (Vard.): $50 \mu \mathrm{M}$, TAK-063: $0.15 \mu \mathrm{M}$, zaprinast (Zapr.): $40 \mu \mathrm{M}$, IBMX: $100 \mu \mathrm{M}$, milrinone (Milr.): $20 \mu \mathrm{M}$, BAY 60-7550: 10 $\mu \mathrm{M}$, sildenafil: $100 \mu \mathrm{M}$. (I) Determination of cAMP concentration of YFP-BeCyclop-expressing BM-derived MKs after incubation with forskolin or illumination with green light for $5 \mathrm{~min}(\mathrm{n}=3)$. 
A

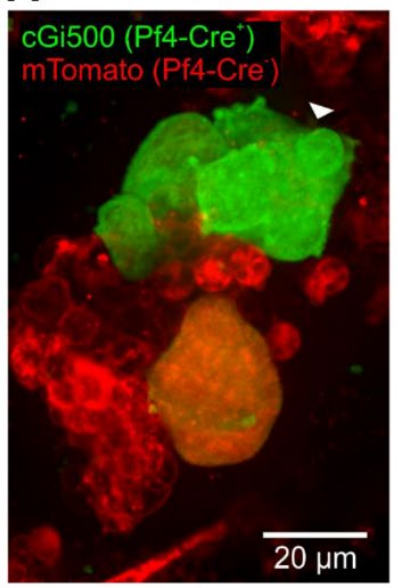

B

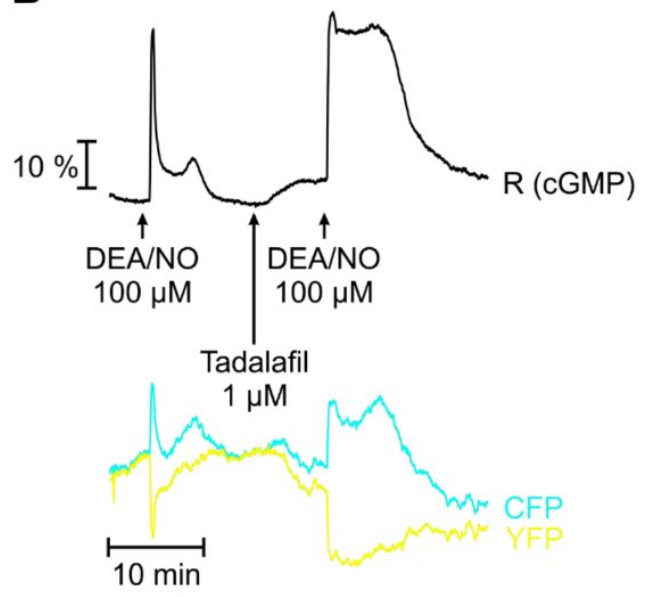

C

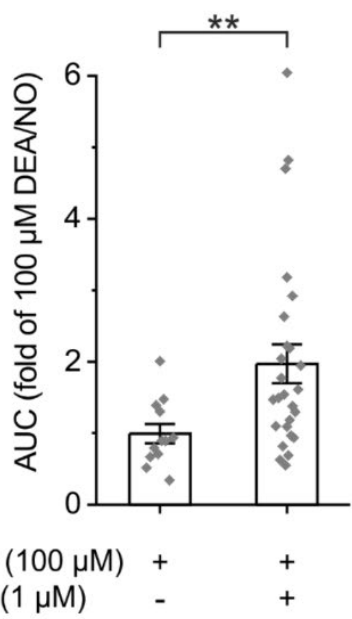

Figure 2. Real-time cGMP imaging in MKs. FRET-based cGMP imaging was performed with BM from MK/platelet-specific cGMP sensor mice. (A) Representative field of view of a measurement. Green color indicates cGMP sensor expressing MKs. mTomato (red) is expressed in all other cells. The white arrowhead points towards the MK measured in (B). (B) Representative real-time FRET/cGMP measurement. During recording, DEA/NO (100 $\mu \mathrm{M})$ was applied for $2 \mathrm{~min}$ with or without $7 \mathrm{~min}$ pre-incubation with tadalafil $(1 \mu \mathrm{M})$. Black trace represents the CFP/YFP ratio $R$, which indicates cGMP concentration changes. Relative fluorescence changes of the individual fluorescent proteins are shown as cyan (CFP) and yellow (YFP) trace. (C) Statistical analysis was performed with the area under the curve (AUC) of cGMP signals induced by DEA/NO $(100 \mu \mathrm{M})$ in the presence of tadalafil $(1 \mu \mathrm{M})$ or vehicle (DMSO; $0.002 \%$ ). Data represent mean \pm SEM of $n \geq 12$ MKs from $\geq 3$ mice. Scale bar, 20 $\mu \mathrm{m}$. 